\title{
Role of surface passivation on visible and infrared emission of Ge quantum dots formed by dewetting
}

\author{
M AOUASSA $^{\mathbf{1}}$, M A ZRIR ${ }^{2, *}$, I JADLI $^{1}$, L S HASSAYOUN $^{1}$, R MGHAIETH $^{\mathbf{1}}$, H MAAREF $^{1}$, \\ L FAVRE $^{3}$, A RONDA ${ }^{3}$ and I BERBEZIER ${ }^{3}$ \\ ${ }^{1}$ Department of Physics, Laboratory of Micro-Opto-Electronic and Nanostructures (LMON), Faculty of Sciences, \\ Monastir 5019, Tunisia \\ ${ }^{2}$ Department of Physics, Atomic Energy Commission of Syria (AECS), Damascus 6091, Syria \\ ${ }^{3}$ IM2NP Faculté des Sciences et Techniques, Campus de Saint Jérôme, Marseille, Cedex 20 13397, France \\ *Author for correspondence (pscientific33@aec.org.sy)
}

MS received 4 March 2018; accepted 24 September 2018; published online 6 March 2019

\begin{abstract}
The dual action of oxide-related defects in the visible and infrared emission of germanium (Ge) self-assembled quantum dots (QDs) is discussed. The Ge particles were fabricated by solid-state dewetting on a thin layer of $\mathrm{SiO}_{2}$. Subsequent surface passivation by amorphous silicon was carried out for several samples. All samples were encapsulated by $\mathrm{SiO}_{2}$. Atomic force microscopy analysis indicates a linear relationship between the size of QDs and the initial thickness of the amorphous Ge films. The crystallization of the QDs was evidenced by transmission electron microscopy and Raman spectroscopy. Photoluminescence measurements show that the main visible emission is blue-green centred around $520 \mathrm{~nm}$. The luminescence attributed to the radiative recombination of quantum-confined excitons is only observed when the surface is in-situ passivated prior to the deposition of the oxide matrix. The results of this work are helpful for optimizing the performance of the optoelectronic devices based on the infrared emission of Ge nanocrystals.
\end{abstract}

Keywords. Solid-state dewetting; Ge quantum dots; oxide-related defects; photoluminescence.

\section{Introduction}

The possibility of tuning the bandgap of low-dimensional semiconductor structures has motivated numerous experimental investigations due to its promising applications in optoelectronics technology [1-5]. Germanium (Ge) nanocrystals (NCs) are of particular interest not only as complementary metal-oxide-semiconductor compatible material but also because of the large effective Bohr radius of excitons as compared to silicon [6], which induces stronger quantum confinement effects on the emission wavelength. For light emitting devices, electrical pumping is crucial. The potential applications of Ge quantum structures as light emitters are, thus, associated with the ability of yielding high emission efficiency due to radiative recombination in the Ge crystal. The origin of light emission from Ge nanostructures is, however, a subject of controversial interpretation of experimental evidence, which is attributed to quantum confinement effects $[4,7,8]$ as well as to defects [9-11]. The evolution of the $\mathrm{Ge}$ electronic structure upon reducing the size was experimentally deduced from X-ray absorption spectra by Bostedt et al [12]. Their results supported the photoluminescence (PL) shifts observed in the near-infrared region [7]. Niquet et al [13] combined ab initio calculations with experimental data and differentiated between two emission ranges: (1) a blue-green emission, which is found to be size-independent; (2) a near-infrared emission arising from the Ge crystal, whose energy is strongly connected to the NC size. The highest PL peak energy that could be assigned to radiative recombination of quantum-confined excitons in Ge was in the red spectral region, for particle sizes of $1 \mathrm{~nm}[7,14-16]$. For higher emission energies, ultra-small $\mathrm{NCs}$ (diameter $<1 \mathrm{~nm}$ ) are required. It is worth noting that the calculated bandgap for such clusters would exhibit emission considerably higher in energy than those observed experimentally $[13,17,18]$. The bluegreen luminescence was experimentally observed from $\mathrm{Ge}$ nanostructures prepared by several methods, such as radiofrequency sputtering [19], stain etching [20], ion implantation/annealing [21], chemical vapour deposition (CVD) growth [11,22] and electrochemical deposition [23]. Ge nanodots can also be fabricated using a dewetting mechanism for thin Ge films on insulator layers [2,24-27]. The sizes of particles produced by the mentioned techniques ranged from $\sim 1 \mathrm{~nm}$ up to the micrometric scale. Similar emissions of these particles cannot be consistent with the size-dependent quantum confinement effects predicted for Ge NCs. It was, thus, commonly attributed to oxide-related defects located either in $\mathrm{GeOx}$, such as oxygen vacancies $\left[\mathrm{VO}^{-}\right]$and oxygen-germanium vacancy pairs $\left[(\mathrm{VGe}, \mathrm{VO})^{+}\right]$ [28-30], or at the interface between $\mathrm{Ge}$ and the oxide matrices [7,11,31]. This emission band was also observed for 
smooth Ge surfaces [32], indicating that even a thin native oxide layer can be a substantial source of the blue-green luminescence.

We fabricated Ge quantum dots (QDs) on a thin $\mathrm{SiO}_{2}$ layer by in-situ dewetting of amorphous Ge films grown by high precision solid source molecular beam epitaxy (SSMBE). The sizes and the densities of QDs can be controlled through the thickness of the amorphous Ge layer. Ge QD surfaces are insitu passivated by an ultra-thin layer of amorphous $\mathrm{Si}$. We compared the emission properties of passivated and unpassivated QDs and we show that the presence of the oxide-related defects is not only accompanied with the visible emission, but also with quenching the infrared emission resulted from band-to-band radiative recombination inside the Ge QDs.

\section{Experimental}

After the formation of a thin layer of thermal $\mathrm{SiO}_{2}$ by a rapid thermal oxidation process on a $\mathrm{Si}(001)$ wafer, the substrates were transferred to the deposition chamber, where a short time annealing was carried out at $750^{\circ} \mathrm{C}$. Then, very-high pure amorphous Ge layers were grown at room temperature by high precision SSMBE system under background pressure of $10^{-10}$ Torr. Ge QDs are formed by dewetting of the amorphous Ge layers. The process involves an in-situ annealing step that results in the development of randomly distributed 3D Ge QDs with very high density. For several samples, the QD formation is followed by in-situ capping with $1 \mathrm{~nm}$ of amorphous silicon for passivation. All samples were subsequently encapsulated by $50 \mathrm{~nm}$ of $\mathrm{SiO}_{2}$ deposited by plasma-enhanced CVD. The fabrication process for $\mathrm{Ge}$ QDs is schematically presented in figure 1.

Size and density distributions of the Ge QDs on $\mathrm{SiO}_{2}$ were studied by atomic force microscopy (AFM) in the tapping mode. Structural analysis of the QDs was achieved by means of high-resolution transmission electron microscopy (HRTEM) using a Jeol 2000FX microscope. Raman measurements, using RAMAN Horiba Jobin-Yvon ARAMIS with spectral resolution of $0.2 \mathrm{~cm}^{-1}$ and diffraction gratings of $1800 \mathrm{~g} \mathrm{~mm}^{-1}$, were performed at room temperature. The formation of pure Ge QDs was evidenced by Raman spectra recorded in the (100) backscattering geometry. The PL of Ge QDs, using Ar-ion laser with an excitation wavelength of $488 \mathrm{~nm}$, was investigated to examine their optical properties and to highlight the role of the amorphous silicon passivation. The PL detection was done using a GaAs detector for the visible range and an InGaAs detector for the infrared range. All PL spectra were recorded at $8 \mathrm{~K}$.

\section{Results and discussion}

Ge QDs were fabricated starting from thin, amorphous Ge films grown on $\mathrm{SiO}_{2} / \mathrm{Si}$ substrate. Solid-state dewetting was observed after in-situ thermal annealing at $550^{\circ} \mathrm{C}$ for $30 \mathrm{~min}$. The size and the density distributions were determined from AFM images for several film thicknesses. Figure 2 displays two examples of the morphology evolution after the dewetting process. Hemispherical QDs are developed with aspect ratios of nearly 1 and with a very high density $\left(>10^{11}\right.$ QDs $\left.\mathrm{cm}^{-2}\right)$.

The statistical analysis indicates that the diameter $(\Phi)$ of the QDs depends only on the initial thickness $(\tau)$ of the amorphous Ge layer, as shown in figure 3. The QD size can, thus, be tuned with a nanoscale precision by varying the thickness of Ge films. The size of the Ge QDs corresponds to a minimum free energy for the $\mathrm{Ge} / \mathrm{SiO}_{2}$ system, which depends on the quantity of Ge deposited on $\mathrm{SiO}_{2}$, as reported by Szkutnik et al [33]. The density of QDs is found to be inversely proportional to $\tau^{2}$.

Cross-sectional HRTEM images of Ge QDs, resulting from dewetting of 1 and $2 \mathrm{~nm}$ amorphous Ge layers, are shown in figure $4 \mathrm{a}$ and $\mathrm{c}$. The formation of the QDs is followed by an amorphous Si capping (1 nm) and final encapsulation with $\mathrm{SiO}_{2}(50 \mathrm{~nm})$. Figure $4 \mathrm{~b}$ and d reveals the crystallization of these hemispherical QDs.

The crystallization of Ge QDs can also be illustrated by Raman spectroscopy. Figure 5 shows Raman spectra obtained for the samples containing Ge QDs with two average diameters (7 and $14 \mathrm{~nm}$ ). The Raman peaks related to the $\mathrm{Ge}-\mathrm{Ge}$ vibrations (about $303 \mathrm{~cm}^{-1}$ ) are observed for the two samples. However, Si-Ge modes [34,35] were not

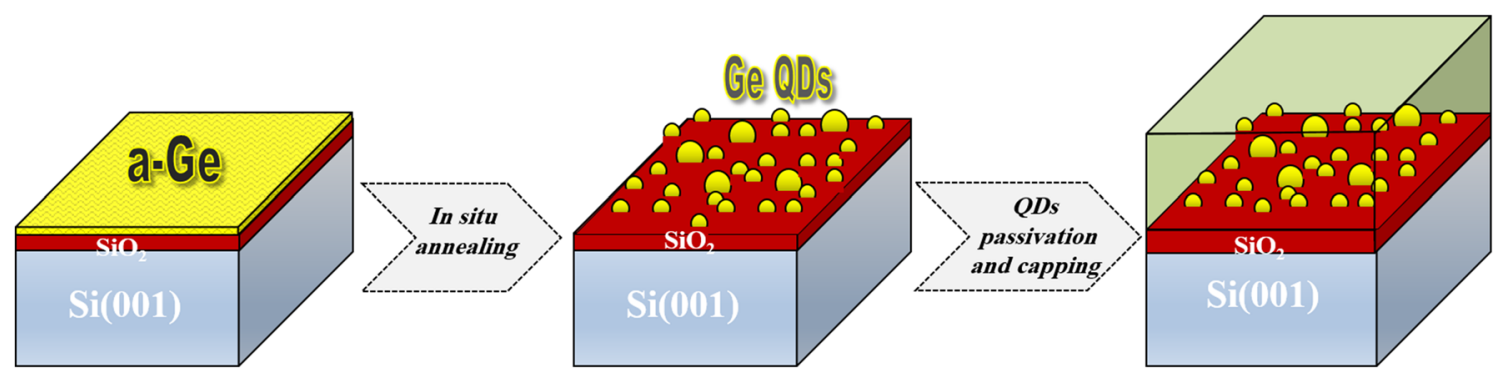

Figure 1. Flowchart of the Ge QD elaboration steps: (a) deposition of amorphous Ge thin layer, (b) formation of Ge QDs by in-situ dewetting at $550^{\circ} \mathrm{C}$ for $30 \mathrm{~min}$, (c) in-situ passivation by $1 \mathrm{~nm}$ of amorphous silicon (optional) and encapsulation with $\mathrm{SiO}_{2}$. 

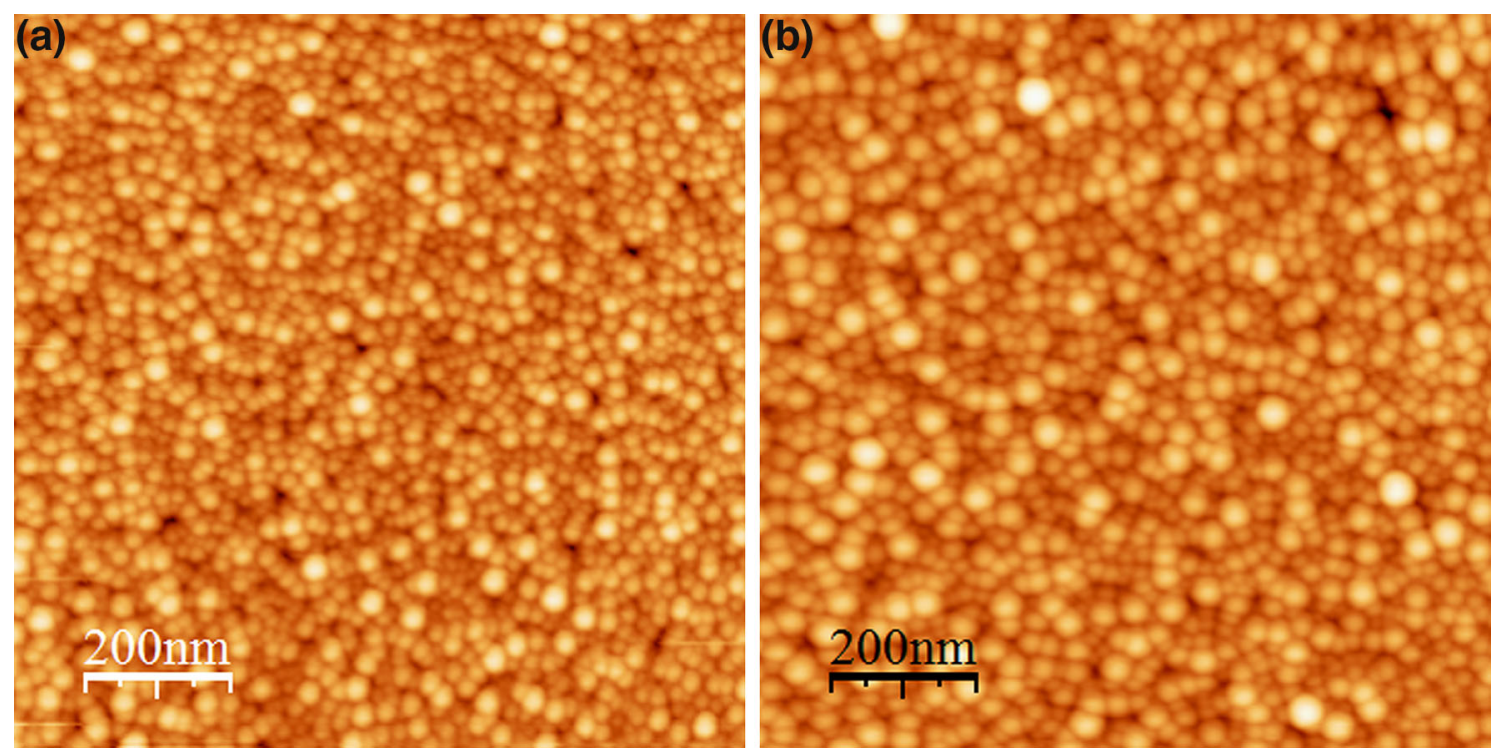

Figure 2. AFM images of Ge QDs with an average diameter of: (a) $7 \mathrm{~nm}$ after dewetting of $1 \mathrm{~nm}$ thick Ge layer and (b) $14 \mathrm{~nm}$ after dewetting of $2 \mathrm{~nm}$ thick Ge layer.
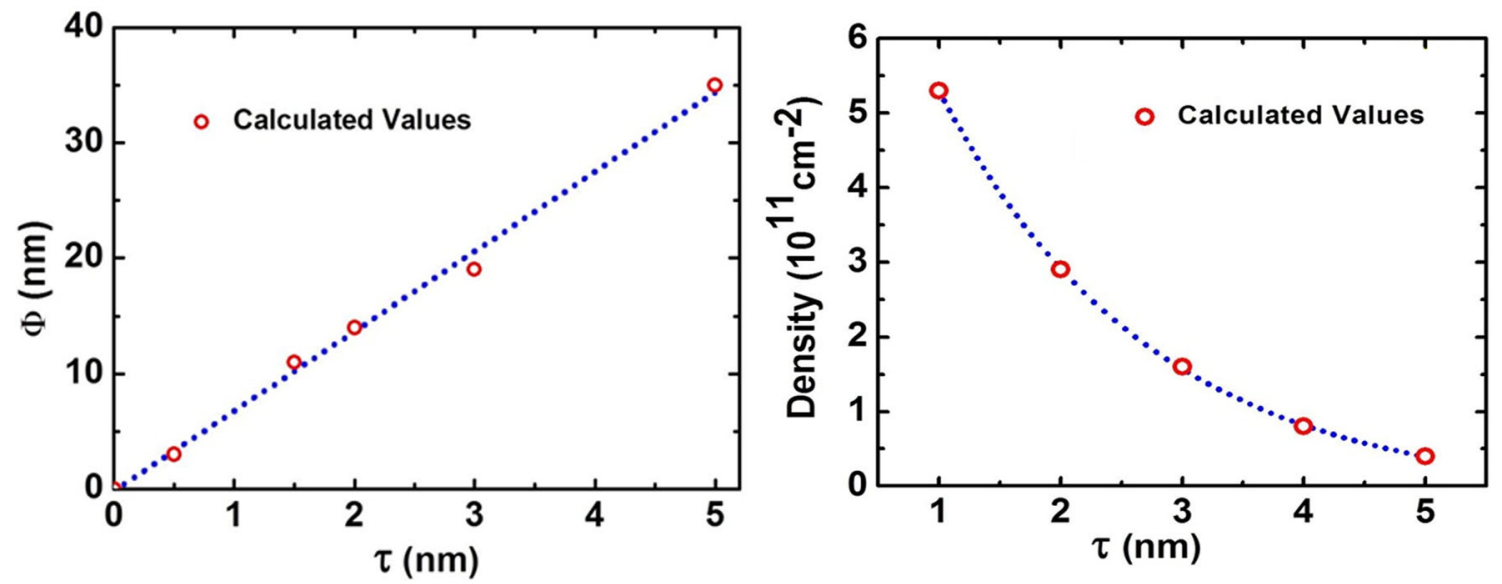

Figure 3. (Left) Evolution of the QD diameter as a function of the thickness ( $\tau$ ). (Right) Evolution of the QD density as a function of $\tau$. The blue dotted lines are a guide to the eye.

detected, which indicates, within the detection limit of our instrument, that there is no interdiffusion between the Ge and Si cap-layer.

The passivation by amorphous silicon aims to prevent the formation of $\mathrm{GeOx}$ at the QD surface, whose contributions are well-established in the visible range. However, despite the passivation, a blue-green luminescence was observed from the samples containing Ge QDs of both sizes ( 7 and $14 \mathrm{~nm}$ ), as shown in figure 6. More interestingly, the same emission was also recorded without Si-passivation. Note that the PL peaks should be located at shorter wavelengths and estimated to be around $500 \mathrm{~nm}$. The drop of the PL intensity at $525 \mathrm{~nm}$ is due to the long pass filter cutoff. The observed emission can be explained by the presence of luminescent-defect-centres related to $\mathrm{GeOx}$ and $\mathrm{SiOx}$ at the interface between the $\mathrm{Ge}$ QDs and the substrate [7,9,11,28,29,31,35].

The excitons generated in the Ge QDs could also diffuse out towards oxide-related states, where they recombine radiatively leading to visible luminescence [36], as it was also suggested to explain the visible emission of porous Si [37]. Indeed, this mechanism causes a deficit of electron-hole pairs, whose recombination results in the infrared emission of $\mathrm{Ge}$ QDs. In the following, we investigate the role of passivation in the infrared PL properties. Without Si-passivation, no emission was measured from the $\mathrm{Ge} Q \mathrm{QD}$ in $\mathrm{SiO}_{2}$ matrix regardless of their diameter, as can be seen in figure 7. However, for passivated QDs, a size-dependent PL shift was observed. The emission of QDs with average sizes of 7 and $14 \mathrm{~nm}$ exhibits 

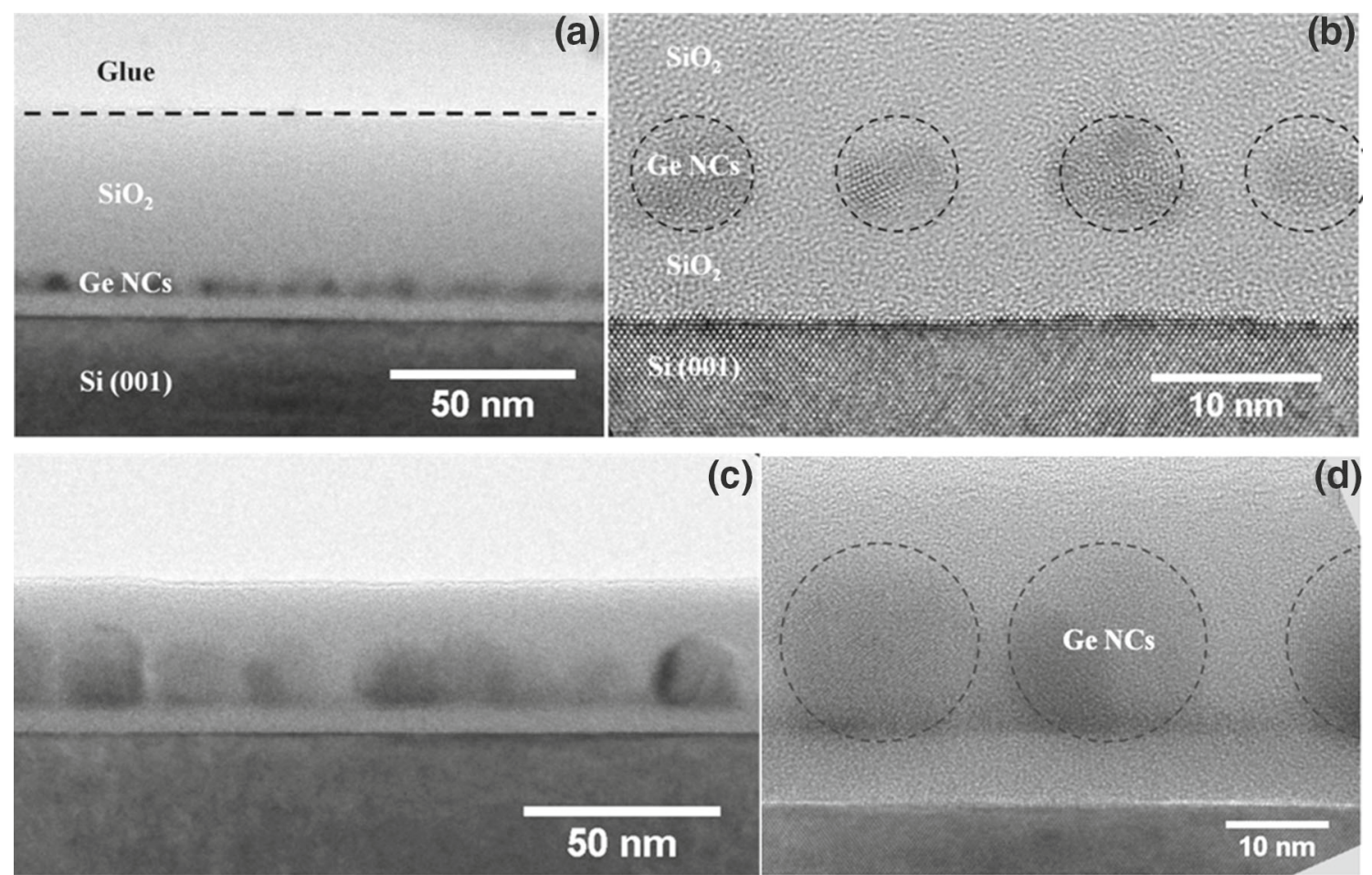

Figure 4. Cross-sectional TEM images after dewetting of Ge films of thicknesses of: (a) $1 \mathrm{~nm}$ and (c) $2 \mathrm{~nm}$. Highresolution images showing the resulted Ge QDs with an average diameter of: (b) $7 \mathrm{~nm}$ and (d) $14 \mathrm{~nm}$.

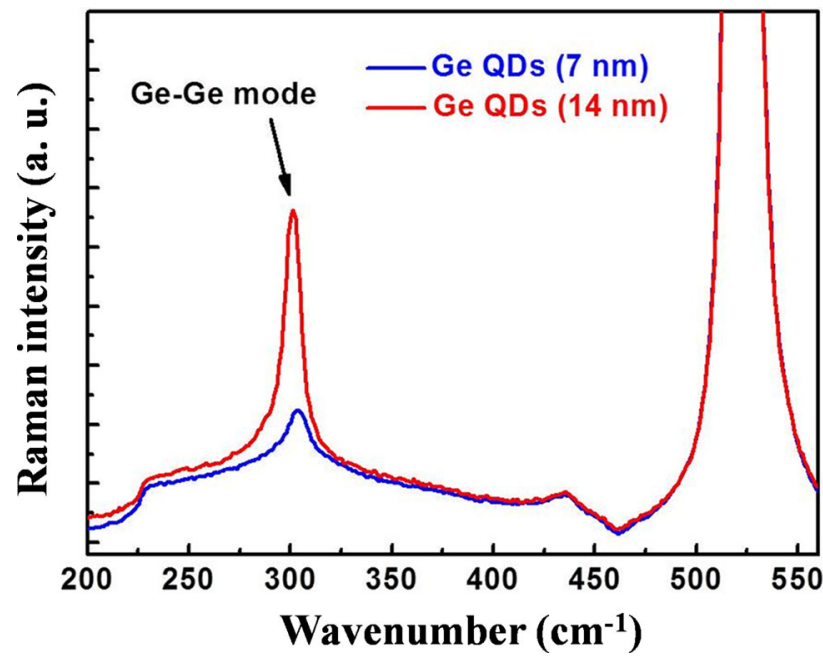

Figure 5. Room temperature Raman spectra showing the $\mathrm{Ge}-\mathrm{Ge}$ mode related to the Ge QDs of two different average diameters $7 \mathrm{~nm}$ (blue) and $14 \mathrm{~nm}$ (red).

broad PL peaks centred at 0.85 and $0.82 \mathrm{eV}$, respectively. These energies are higher than the bandgap of $\mathrm{Ge}(0.74 \mathrm{eV})$. The shifts from the bulk value can uniquely be related to confinement effects, since the QDs are fully relaxed, as indicated by Raman measurements. The PL width depends on the QD size distribution. It is noteworthy that similar emissions

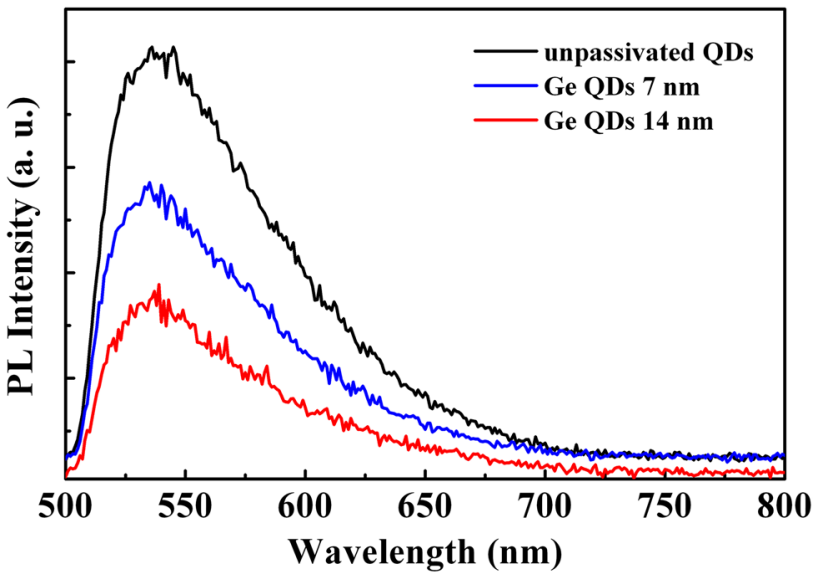

Figure 6. PL spectra exhibiting the same emission energy for $\mathrm{Ge}$ QDs, embedded in $\mathrm{SiO}_{2}$ matrix of different average sizes.

were also observed for Ge QDs epitaxially grown on silicon [38-40].

Although the passivation is not complete, as one side of the QDs is always in contact with $\mathrm{SiO}_{2}$, we find, nevertheless, that it reduces the loss of the photogenerated electron-hole pairs that is induced by the defect-related states at the QD surface or in the surrounding oxides. 


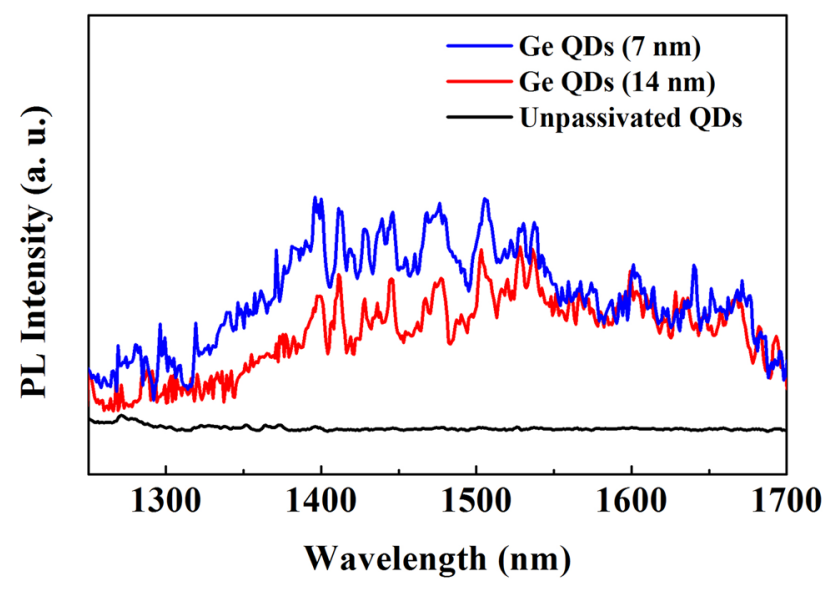

Figure 7. PL spectra of Si-passivated Ge QDs of different average diameters: $7 \mathrm{~nm}$ (blue) and $14 \mathrm{~nm}$ (red). The black spectrum for the unpassivated QDs is included for comparison.

\section{Conclusion}

Self-assembled Ge QDs were fabricated by dewetting of thin, amorphous Ge layers on $\mathrm{SiO}_{2}$. The crystallization of the Ge QDs was confirmed by TEM and Raman spectroscopy. The QDs were found to be fully relaxed. While it is well-established that the presence of oxide-related defects promotes a radiative pathway for the generated carriers in $\mathrm{GeOx}$, our measurements reveal a dual role of the oxiderelated defects, which appears in: (a) the blue-green emission band and (b) quenching the infrared emission resulted from radiative recombination inside the Ge QDs. Surface passivation allows for the quantum-confined excitons to recombine radiatively inside the Ge NCs resulting in infrared emission, which is very important for optoelectronic applications and long-haul telecommunications.

\section{Acknowledgement}

One of the authors (M A Zrir) thanks Prof I Othman, the Director General of the AECS, for his ongoing support.

\section{References}

[1] Canham L T 2000 Nature $\mathbf{4 0 8} 411$

[2] Kanoun M, Lemiti M, Bremond G, Souifi A, Bassani F and Berbezier I 2004 Superlattices Microstruct. 36143

[3] Bouchier D, Yam V, Halbwax M, Nguyen L, Debarre D and Fossard F 2006 ECS Trans. 3569

[4] Lee D C, Pietryga J M, Robel I, Werder D J, Schaller R D and Klimov V I 2009 J. Am. Chem. Soc. 1313436

[5] Zwanenburg F A, Dzurak A S, Morello A, Simmons M Y, Hollenberg L C L, Klimeck G et al 2013 Rev. Mod. Phys. 85 961

[6] Efros A L and Rosen M 2000 Annu. Rev. Mater. Sci. 30475
[7] Takeoka S, Fujii M, Hayashi S and Yamamoto K 1998 Phys. Rev. B $\mathbf{5 8} 7921$

[8] Wilcoxon J P, Provencio P P and Samara G A 2001 Phys. Rev. B 64035417

[9] Min K S, Shcheglov K V, Yang C M, Atwater H A, Brongersma M L and Polman A 1996 Appl. Phys. Lett. 682511

[10] Rebohle L, Gebel T, von Borany J, Skorupa W, Helm M, Pacifici D et al 2002 Appl. Phys. B 7453

[11] Avella M, Prieto Á C, Jiménez J, Rodríguez A, Sangrador J, Rodríguez T et al 2008 Mater. Sci. Eng. B 147 200

[12] Bostedt C, Van Buuren T, Willey T M, Franco N, Terminello L J, Heske C et al 2004 Appl. Phys. Lett. 8420

[13] Niquet Y M, Allan G, Delerue C and Lannoo M 2000 Appl. Phys. Lett. 771182

[14] Kartopu G, Sapelkin A V, Karavanskii V A, Serincan U and Turan R 2008 J. Appl. Phys. 103113518

[15] Chang S-T and Liao S-H 2009 J. Vac. Sci. Technol. B 27 535

[16] Fraj I, Favre L, David T, Abbarchi M, Liu K, Claude J B et al 2017 Appl. Surf. Sci. 419476

[17] Takagahara T and Takeda K 1992 Phys. Rev. B 4615578

[18] Maeda Y 1995 Phys. Rev. B 511658

[19] Okamoto S and Kanemitsu Y 1996 Phys. Rev. B 5416421

[20] Kartopu G, Bayliss S C, Karavanskii V A, Curry R J, Turan R and Sapelkin A V 2003 J. Lumin. 101275

[21] Singha A, Roy A, Kabiraj D and Kanjilal D 2006 Semicond. Sci. Technol. 211691

[22] Ko T S, Shieh J, Yang M C, Lu T C, Kuo H C and Wang S C 2008 Thin Solid Films 5162934

[23] Jawad M J, Hashim M R, Ali N K, Corcoles E P and Arora Vijay K 2014 J. Electochem. Soc. 161 D801

[24] Kanjilal A, Lundsgaard Hansen J, Gaiduk P, Nylandsted Larsen A, Cherkashin N, Claverie A et al 2003 Appl. Phys. Lett. 82 1212

[25] Berbezier I, Karmous A, Ronda A, Sgarlata A, Balzarotti A, Castrucci P et al 2006 Appl. Phys. Lett. 89063122

[26] Szkutnik P D, Sgarlata A, Motta N, Placidi E, Berbezier I and Balzarotti A 2007 Surf. Sci. 6012778

[27] Rowell N L, Lockwood D J, Karmous A, Szkutnik P D, Berbezier I and Ronda A 2008 Superlattices Microstruct. 44 305

[28] Sendova-Vassileva M, Tzenov N, Dimova-Malinovska D, Rosenbauer M, Stutzman M and Josepovits K V 1995 Thin Solid Films 255282

[29] Zacharias M and Fauchet P M 1997 Appl. Phys. Lett. 71 380

[30] Viswanathamurthi P, Bhattarai N, Kim H Y, Khil M S, Lee D R and Suh E-K 2004 J. Chem. Phys. 121441

[31] Das S, Singha R K, Gangopadhyay S, Dhar A and Ray S K 2010 J. Appl. Phys. 108053510

[32] Zrir M A, Saloum S, Alkhaled B and Shaker S A 2017 Surf. Sci. 6595

[33] Szkutnik P D, Karmous A, Bassani F, Ronda A, Berbezier I, Gacem K et al 2008 Eur. Phys. J. Appl. Phys. 41103

[34] Perova T S, Wasyluk J, Lyutovich K, Kasper E, Oehme M, Rode K et al 2011 J. Appl. Phys. 109033502

[35] Wu X L, Gao T, Siu G G, Tong S and Bao X M 1999 Appl. Phys. Lett. 742420

[36] Nozaki S, Sato S, Denda A, Ono H and Morisaki H 1994 MRS Proc. 358133 
[37] Qin G G and Jia Y Q 1993 Solid State Commun. 86559

[38] Loo R, Meunier-Beillard P, Vanhaeren D, Bender H, Caymax M, Vandervorst W et al 2001 J. Appl. Phys. 902565
[39] Dashiell M W, Denker U, Muller C, Costantini G, Manzano C, Kern K et al 2002 Appl. Phys. Lett. 801279

[40] Wang K L, Cha D, Liu J and Chen C 2007 Proc. IEEE 951866 